[18] N. Franceschini, J. Pichon, C. Blanes, and J. M. Brady, "From insect vision to robot vision," Philos. Trans.: Biol. Sci., vol. 337, no. 1281, pp. 283-294, 1992.

[19] T. Collett, "Insect vision: Controlling actions through optic flow," Current Biol., vol. 12, pp. R615-R617, 2002.

[20] N. Takeda, M. Watanabe, and K. Onoguchi, "Moving obstacle detection using residual error of FOE estimation," in Proc. IEEE/RSJ Int. Conf. Intell. Robot. Syst., 1996, pp. 1642-1647.

[21] L. Kaufman, Sight and Mind. New York: Oxford Univ. Press, 1974.

[22] C. Shannon, "A mathematical theory of communication," Bell Syst. Tech. $J .$, vol. 27, pp. 379-423-623-656, 1948.

[23] M. Varma and A. Zisserman, "Texture classification: Are filter banks necessary?," in Proc. IEEE Comput. Soc. Conf. Comput. Vis. Pattern Recog., 2003, vol. 2, pp. 691-698.

[24] T. Kohonen, Self-Organizing Maps. New York: Springer-Verlag, 2001

[25] G. de Croon, E. de Weerdt, C. de Wagter, B. Remes, and R. Ruijsink, "The appearance variation cue for obstacle avoidance," in Proc. IEEE Int. Conf. Robot. Biomimetics, 2010, pp. 1606-1611.

[26] B. Lucas and T. Kanade, "An iterative image registration technique with an application to stereo vision," in Proc. Imag. Understand. Workshop, 1981, pp. 121-130.

[27] J.-Y. Bouguet, "Pyramidal implementation of the Lucas Kanade feature tracker: Description of the algorithm," Microprocessor Research Lab, Intel Corp., Santa Clara, CA, Tech. Rep. CMU-RI-TR-02-16, 2000.

[28] T. Fawcett, "An introduction to ROC analysis," Pattern Recog. Lett., vol. 27, pp. 861-874, 2006.

\section{A Comparative Evaluation of Control Interfaces for a Robotic-Aided Endoscopic Capsule Platform}

Gastone Ciuti, Marco Salerno, Gioia Lucarini, Pietro Valdastri, Alberto Arezzo, Arianna Menciassi, Mario Morino, and Paolo Dario

\begin{abstract}
Wireless capsule endoscopy offers significant advantages compared with traditional endoscopic procedures, since it limits the invasiveness of gastrointestinal tract screening and diagnosis. Moreover, active locomotion devices would allow endoscopy to be performed in a totally controlled manner, avoiding failures in the correct visualization of pathologies. Previous works demonstrated that magnetic locomotion through a robotic-aided platform would allow us to reach this goal reliably. In this paper, the authors present a comparative evaluation of control methodologies and user interfaces for a robotic-aided magnetic platform for capsule endoscopy, controlled through human-robot cooperative and teleoperated control algorithms. A detailed statistical analysis of significant control parameters was performed: teleoperated control is the more reliable control approach, and a serial kinematic haptic device results as the most suitable control interface to perform effective robotic-aided endoscopic procedures.
\end{abstract}

Manuscript received May 4, 2011; accepted November 11, 2011. Date of publication December 23, 2011; date of current version April 9, 2012. This paper was recommended for publication by Associate Editor M. Sitti and Editor B. J. Nelson upon evaluation of the reviewers' comments. This work was supported in part by the European Commission in the framework of the VECTOR FP6 European project EU/IST-2006-033970 and ARAKNES FP7 European project EU/IST-2008-224565.

G. Ciuti, M. Salerno, G. Lucarini, A. Menciassi, and P. Dario are with The BioRobotics Institute, Scuola Superiore Sant' Anna, 56025 Pontedera, Pisa, Italy (e-mail: g.ciuti@sssup.it; m.salerno@sssup.it; g.lucarini@sssup.it; arianna@sssup.it; paolo.dario@sssup.it).

P. Valdastri is with Vanderbilt University, Nashville, TN 37235-1592 USA (e-mail: p.valdastri@vanderbilt.edu).

A. Arezzo and M. Morino are with the Digestive, Colorectal, and Minimal Invasive Surgery, University of Torino, 10124 Torino, Italy (e-mail: alberto.arezzo@mac.com; mario.morino@unito.it).

Color versions of one or more of the figures in this paper are available online at http://ieeexplore.ieee.org.

Digital Object Identifier 10.1109/TRO.2011.2177173
Index Terms-Capsule endoscopy, endoscopic robotic platform, telerobotics, virtual reality and interfaces.

\section{INTRODUCTION}

In medicine, physicians are faced with several natural limitations that can be sometimes overcome by the use of robotics [1]. For this reason, over the past decade, medical robotics has grown to be an exciting new field within general medicine and surgery [2], [3]. Above all, robotics can standardize procedures making tasks less dependent upon medical users' skills and has the potential to improve not only surgical applications, but also diagnostic procedures, such as those conducted in the endoscopic field, thanks to high precision and predictability [4], [5].

Beginning in the 1990s, there has been increasing activity in the application of robotic technologies to improve endoscopic procedures in the gastrointestinal (GI) tract [6], [7]. The objective of robotic colonoscopy and gastroscopy is to obtain more effective diagnoses, reduce pain in patients, and make diagnostic procedures more uniform, independently of the endoscopist's manual abilities. In this framework, one of the main issues that arises in the exploitation of a robotic approach, alongside preserving the intuitiveness of traditional procedures, is how the surgeon or physician can control the robotic system.

\section{A. Control Methodologies for Endoscopic Capsules With Magnetic Locomotion}

An ideal control methodology, which is also interfaced with a dedicated user interface, should allow the physician to command a slave robotic system as naturally as possible, by performing the same movements and gestures, and even holding the same operational tools [8].

In this framework, control methodologies and user interfaces have been investigated in order to select the optimal solution for the control of a magnetic locomotion robotic platform for endoscopic capsule procedures, which are developed by the authors and described in detail in [9] and [10]. This study describes and compares a human-robot cooperative (HRC) and teleoperated (TO) control methodologies and their relative user interfaces. As summarized in Fig. 1, knob-based and serial kinematic haptic user interfaces (SKUIs) were selected within the TO control representing, together with HRC control, two control approaches, as well as three potential solutions to control the robotic system. This study provides a quantitative statistical analysis of relevant control parameters during a typical endoscopic task to control the magnetic locomotion of an endoscopic capsule.

In particular, the HRC control strategy was implemented, exploiting a force/torque $(\mathrm{F} / \mathrm{T})$ sensor mounted at the robot distal tip as the active user interface. The HRC control methodology has the significant advantage of combining the intuitiveness of a manual procedure with the accuracy provided by the robotic arm. Movements that are imposed by the user on the robot end effector (EE) can be properly scaled and tremor potentially compensated. Above all, the physician is completely aware of the surrounding environment, since he is in direct contact with the patient [11].

On the other hand, the TO procedure enables, in principle, remote performance of tasks and allows the robot to be transparent to the operator. As a result, the medical doctor can control the movement of the robotic system, via a remote user interface, using only images and/or sensorial feedback received from the EE and/or operating room environment. The TO control has the disadvantage that the physician loses direct contact with the patient and the system. Therefore, the performance of a reliable medical procedure requires mental abstraction by the medical doctor; for this reason, choosing an efficient and userfriendly interface represents a critical point in the design of a TO 


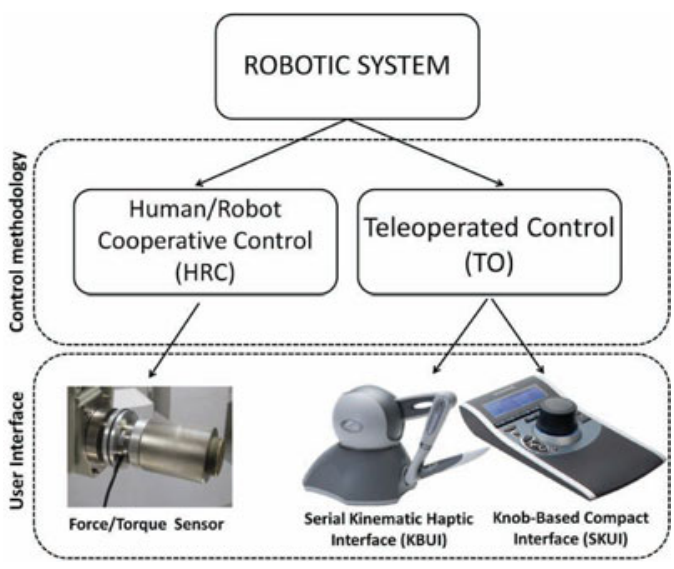

Fig. 1. Control methodologies and selected dedicated interfaces.

robotic system for endoscopy. To address these issues, two different user interfaces were compared for the TO control mode. A 6-degree-offreedom (DOF) knob-based user interface (KBUI) was chosen as possible control peripheral (3-D SpacePilot, 3Dconnexion Inc., Waltham, MA) given its compact and defined operating workspace. The second selected TO control interface was a 6-DOF SKUI (Phantom Omni, SensAble Tech., Inc., Woburn, MA), whose serial kinematics resembles robotic arm one.

\section{RoBotic Platform OVERVIEW AND CONTROL ARCHITECTURE}

The proposed robotic platform, which is schematically represented in Fig. 2, is composed of a 6-DOF industrial robotic arm capable of moving and orienting an external permanent magnet, a wired magnetic capsule, and a human-machine interface to direct the motion of the robotic arm and displaying real-time sensorial feedback.

Compared with the platform described in [9], robotic arm control algorithms and a dedicated capsule, provided with magnetic link sensor, were developed in this study and are described in detail in the following sections.

\section{A. Robotic System and Motion Control Architecture}

The robotic system, which is depicted in Fig. 2(b), uses a 6-DOF industrial robot RV-3SB and CR2B controller produced by Mitsubishi Electric. Compatibly with the required endoscopic capsule motion in the diagnostic procedure, only 3 DOFs were exploited (1 DOF for the forward/backward EE translation and 2 DOFs for the roll and yaw EE orientation) in order to reduce complexity while performing the entire procedure. These DOF allow us to steer the camera pill with pitch and yaw angles and to move the capsule forward and backward along the colon path. Vertical motion of the EE was not included in this study, as well as in consideration of the substantially 2-D shape of the colon. Vertical position was adjusted at the beginning of the procedure in order to guarantee a stable magnetic link.

A motion control system was developed employing the specific and proprietary real-time architecture of Mitsubishi Electric in order to achieve accurate, smooth, and flexible motion control of the anthropomorphic robotic arm. The control architecture is composed of high-level control (HLC) software written in $\mathrm{C}++$ that establishes bidirectional communication based on the UDP/IP protocol with the robotic arm control unit. The software acquires proportional motion command data, i.e., $o(k) \in \Re\{6\}$ at the control cycle $k$, which is obtained from the control user interfaces, and processes the values exploiting diagonal sensitivity matrices $\mathrm{G} \epsilon \Re\{6 \times 6\}$, resulting in incremental parameters of absolute motion for translational and orientational movements of the EE. In case of HRC control, a forces and torques six-element vector $f(k)$ is replaced with the $o(k)$ vector. The resultant position and orientation incremental values of the EE, i.e., $d p(k) \in \Re\{6\}$, are then added to the previous absolute position and orientation state value $p(k-1)$, and the resultant $p(k)$ absolute position and orientation command data are retrieved by the robot controller at each time step (the motion control cycle lasts $7.1 \mathrm{~ms}$ ):

$$
\left\{\begin{array}{l}
p(k)=p(k-1)+d p(k) \\
d p(k)=G \times o(k) .
\end{array}\right.
$$

The CR2B controller unit implements low-level control software provided by Mitsubishi Electric that waits for absolute position data provided by the HLC software to update the EE position and orientation of each control cycle. After the command value is sent to the servo systems, the EE position and orientation, i.e., $p(k-1)$, and each joint angle, i.e., $j(k-1)$, are sent to the PC and processed by the HLC software in order to constrain the robotic arm motion within the specified robot workspace, to limit the angle ranges and to avoid singularities.

\section{B. Wired Capsule and Sensing Feedback}

The endoscopic capsule that is represented in Fig. 2(a) has a diameter of $15 \mathrm{~mm}$, a length of $45 \mathrm{~mm}$, and weighs $9.6 \mathrm{~g}$. The prototype was developed with the purpose of performing experimental tests, and therefore, its size is not yet compatible with swallowing. The vision system consists of a wired charge-coupled device camera (KARL STORZ $\mathrm{GmbH}$ and Co. KG, Tuttlingen, Germany) and six white light-emitting diodes (NESW007BT, Nichia Chemical Europe GmbH, Nuremberg, Germany). The image stream is transmitted to the human-machine interface and displayed on a dedicated video screen [see Fig. 2(c)]. In addition to diagnosis, this image stream is used to maneuver the capsule along the colon lumen as the main motion control feedback to the operator.

However, vision alone may not be sufficient for the complete control of the capsule in the proposed robotic procedure. In order to address this problem, the implementation of a magnetic link strategy could guarantee a stable, reliable, and effective link between the capsule and the robot $\mathrm{EE}$ and avoid losing the device during the procedure. In fact, once an appropriate magnetic link is guaranteed, the capsule will be correctly aligned with the external magnet, and the operator, by exploiting the implemented rotational matrix of the EE motion with respect to the input reference, will be able to control the capsule by simply imposing motion commands on the user interfaces.

From a technical viewpoint, a custom triaxial magnetic sensor, which is composed of three monoaxial commercially available sensors (Hall Effect Sensor CY-P15A, Chen Yang Technologies GmbH and Co. KG), was integrated into the capsule (total sensor volume of around 200 $\mathrm{mm}^{3}$ ). The sensor outputs were elaborated in real-time with a software routine developed in LabVIEW 8.6 (National Instruments, Inc., Baltimore, MD) that determines the magnetic field module outputs and compares this value with a predefined magnetic field threshold. An auxiliary video screen of the human-machine interface, which is represented in Fig. 2(c), returns the Hall effect sensor feedback via a visual alarm signal and by an additional acoustic signal, informing the user about the internal/external permanent magnets magnetic link stability. Once the user receives this feedback, the optimal link has to be recovered before carrying on with the diagnostic procedures. The external platform view completes the human-machine interface and is shown to the user on the same auxiliary video screen, as represented in Fig. 2(c). 


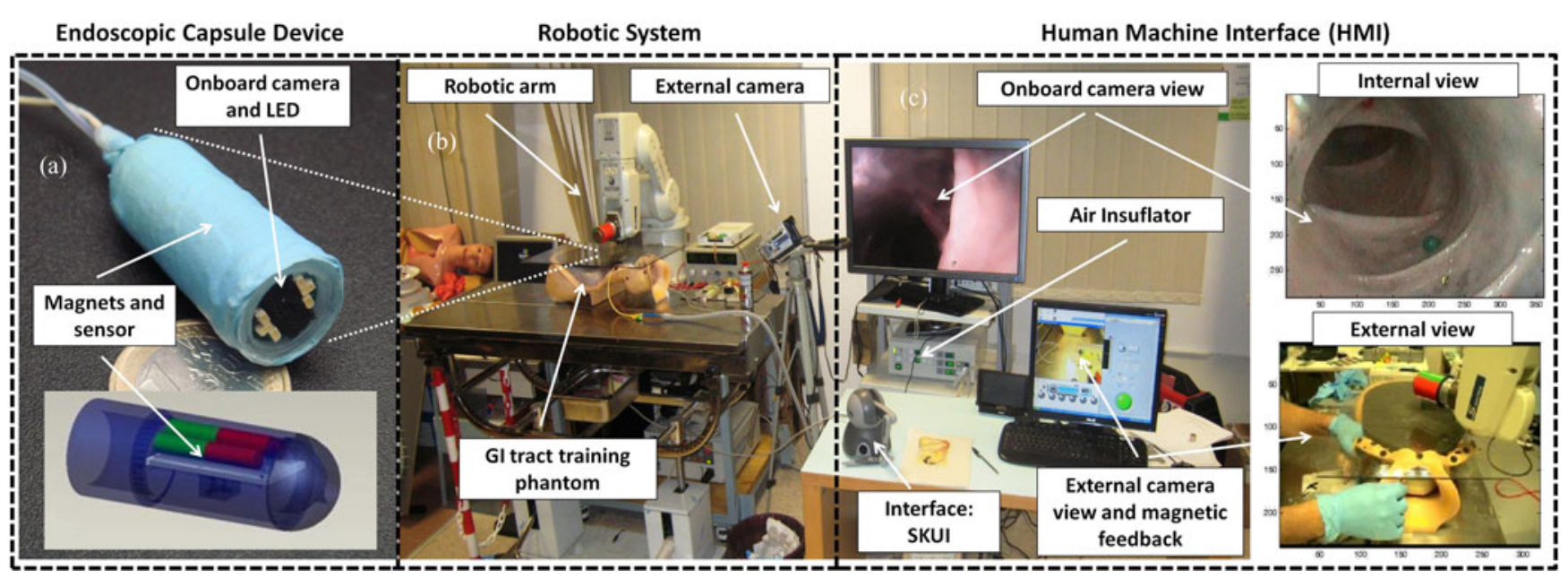

Fig. 2. System architecture of the robotic platform composed of (a) a wired endoscopic capsule, (b) a robotic system, and (c) a human-machine interface.

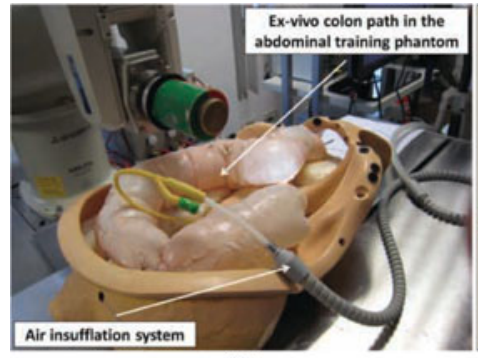

(a)

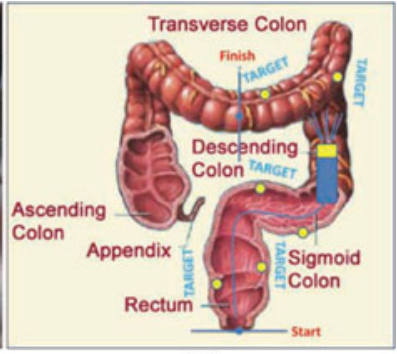

(b)
Fig. 3. (a) Segment of fresh porcine colon attached to the abdominal training phantom. (b) Path of the ex vivo experimental trials.

\section{EXPERIMENTAL METHODS AND RESUltS}

In order to compare performance of the HRC and TO control algorithms and of their user interfaces, an ex vivo experimental protocol was defined and carried out in collaboration with a team of 15 resident well-trained endoscopists by means of a quantitative statistic analysis of relevant control parameters during a typical task of colonoscopy.

\section{A. Experimental Methods}

The proposed task consisted of exploring a segment of ex vivo swine colon composed of straight and curved paths inserted in a human abdominal phantom (Limbs \& Things Ltd., Bristol, U.K.) [see Fig. 3(a)] and arranged to mimic human anatomical features of the entire colon tract, from the rectum to the cecum (approximately $850 \mathrm{~mm}$ in length), also including vertical physiological variations. A 500-mm-long segment was selected for the task; it would be difficult to cover longer segments mainly because the wires of the current camera integrated in the capsule hamper the motion of the device for longer tract.

The experiment was carried out at a fixed constant internal pressure of $1 \mathrm{mmHg}$, maintained by an air insufflator (Surgiflator-40, W.O.M. World of Medicine AG, Germany). Several targets were placed along the internal tissue wall (from eight up to 12 spherical targets, $5 \mathrm{~mm}$ in diameter), and their number and position were randomly changed in each trial to avoid any bias.

The capsule was steered using the robotic-aided approach with the three different interface solutions, according to the HRC and TO control methodology. The goal was to navigate the capsule through the colon, starting from the rectum and reaching the middle of the transverse colon, and to obtain a good view of all targets [see Fig. 3(b)]. The user was asked to identify and assert each target color and to conclude the procedure once completely satisfied with the exploration.

A preliminary pilot test suggested that 45 trials would be enough to guarantee statistical significance. Therefore, all 15 endoscopists were asked to make three test sessions in order to test all three control solutions.

In order to compare the performance of the control solutions, the gain matrices $(\mathrm{G})$ implemented within real-time HRC and TO control were properly tuned to return the same EE output ranges of speed (maximum value of $15 \mathrm{~mm} / \mathrm{s}$ ). The distance between the $\mathrm{EE}$ with the external magnet and the phantom model was maintained constant during all the procedures, at a conservative predefined value (i.e., $100 \mathrm{~mm}$ ) that was chosen to guarantee a reliable magnetic link, and also considering the substantially 2-D shape of the colon. Percentage of identified markers, time needed to execute the test, distance covered by the $\mathrm{EE}$, number of magnetic link losses (\#MLLs), and robot EE average speed parameters were derived and analyzed in detail by means of a statistical analysis with MATLAB (Mathworks, Inc., Natick, MA) statistical routines. Comparisons were made between the two different control methodologies (i.e., HRC and TO control) and among the three different control interfaces, progressively refining the statistical distribution estimation through a bar chart, box-and-whisker diagrams, $t$-test ( $p$-values equal to 0.01 and 0.005 ), and analysis of variance (ANOVA) approaches.

Starting from the assumption that smoother robotic arm motions provide more reliable and efficient control of the capsule in the GI tract and more fluid real-time image stream, the smoothness of the robotic arm EE trajectories was parameterized and considered as a relevant parameter in the evaluation analysis. Considering turbulence on trajectories as a stochastic process depending on the involved control interface, an estimation of the power spectral density of the signal (using a periodogram) was applied on the robotic arm EE X and Y motion trajectories. The frequency corresponding to $50 \%$ of the spectral density area was considered and determined for each executed data-analysis trial for the $\mathrm{X}\left(\mathrm{f}_{s d X}\right)$ and $\mathrm{Y}\left(\mathrm{f}_{s d Y}\right)$ robotic arm movements, and the same statistical analysis was performed on these parameters.

\section{B. Results and Discussion}

All medical doctors successfully completed the ex vivo experimental trials, thus showing promising results in terms of procedure accuracy and reliability (see Fig. 4). 


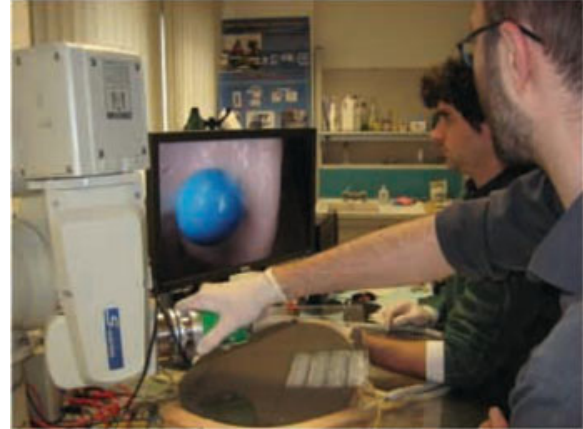

(a)

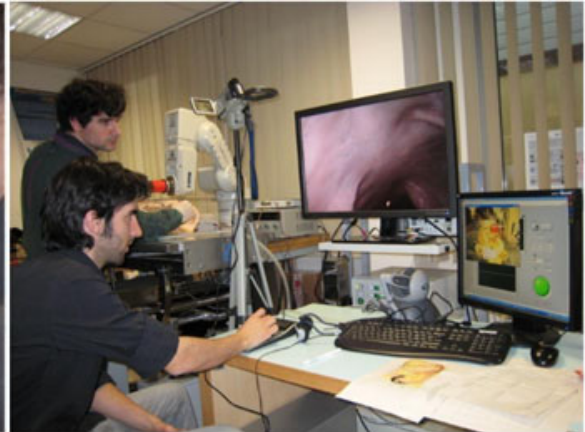

(b)

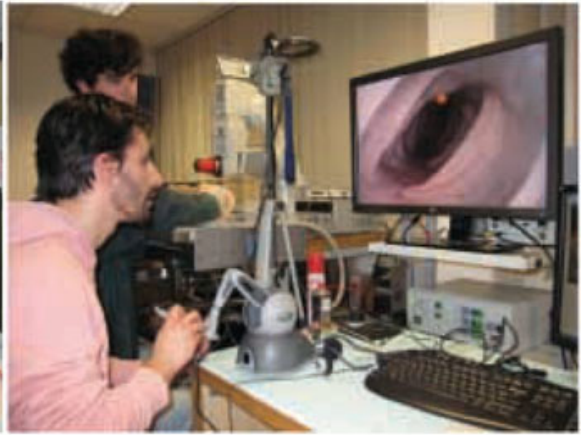

(c)

Fig. 4. Experimental trials performed by medical doctors when controlling the robotic platform by means of (a) HRC control and TO control with the (b) KBUI and with the (c) SKUI.

TABLE I

Mean Value, SSD, ASD, and Range of the Processed Parameters for (a) HRC CONTROL AND TO CONTROL WITH THE (b) KBUI AND (c) SKUI

(a)

\begin{tabular}{|l|l|l|l|l|}
\hline HRC control & Mean & SSD & ASD & Range \\
\hline Marker [\%] & 84.6 & 6.2 & $+6.9 /-6.5$ & $72.7-92.3$ \\
\hline Time [s] & 298 & 95 & $+81 /-69$ & $157-499$ \\
\hline Distance [m] & 2.6 & 1.2 & $+1.1 /-1.2$ & $1-5.2$ \\
\hline \#MLLs & 3.5 & 1.5 & $+1.7 /-1.8$ & $0-6$ \\
\hline EE speed [mm/s] & 8.9 & 3.4 & $+2.9 /-3.5$ & $5.4-16.5$ \\
\hline $\mathbf{f}_{s d X}[\mathbf{m H z}]$ & 8.9 & 4.2 & $+5.2 /-3.5$ & $4.1-16.9$ \\
\hline $\mathbf{f}_{s d Y}[\mathbf{m H z}]$ & 3.7 & 1.3 & $+1.8 /-1.0$ & $1.9-7.1$ \\
\hline
\end{tabular}

(b)

\begin{tabular}{|l|l|l|l|l|}
\hline KBUI TO control & Mean & SSD & ASD & Range \\
\hline Marker [\%] & 79.6 & 11.3 & $+11.2 /-11.7$ & $55.6-92.3$ \\
\hline Time [s] & 440 & 124 & $+148 /-146$ & $264-707$ \\
\hline Distance [m] & 1.3 & 0.4 & $+0.5 /-0.5$ & $0.7-2.0$ \\
\hline \#MLLs & 1.9 & 1.2 & $+1.1 /-0.9$ & $0-4$ \\
\hline EE speed [mm/s] & 3.0 & 0.8 & $+0.6 /-0.6$ & $2.0-5.1$ \\
\hline $\mathbf{f}_{s d X}[\mathbf{m H z}]$ & 5.9 & 2.5 & $+3.2 /-1.9$ & $1.7-11.2$ \\
\hline $\mathbf{f}_{s d Y}[\mathbf{m H z}]$ & 2.5 & 0.7 & $+0.7 /-0.7$ & $1.4-3.6$ \\
\hline
\end{tabular}

(c)

\begin{tabular}{|l|l|l|l|l|}
\hline SKUI TO control & Mean & SSD & ASD & Range \\
\hline Marker [\%] & 79.6 & 10.6 & $+12.8 /-12.2$ & $55.6-90.9$ \\
\hline Time [s] & 573 & 216 & $+172 /-182$ & $280-1006$ \\
\hline Distance [m] & 1.1 & 0.5 & $+0.5 /-0.47$ & $0.4-2.2$ \\
\hline$\#$ MLLs & 2.0 & 1.1 & $+1 /-0.9$ & $0-4$ \\
\hline EE speed [mm/s] & 1.9 & 0.7 & $+0.9 /-0.8$ & $1.1-3.7$ \\
\hline $\mathbf{f}_{s d X}[\mathrm{mHz}]$ & 3.3 & 1.4 & $+1.5 /-1.4$ & $1.2-6.2$ \\
\hline $\mathbf{f}_{s d Y}[\mathbf{m H z}]$ & 1.9 & 0.6 & $+0.5 /-0.6$ & $1.1-2.9$ \\
\hline
\end{tabular}

Regarding the statistical analysis, bar charts (see Table I) did not show significant differences in the percentage of identified marker, elapsed time, distance, and \#MLLs for different control methodologies and interfaces, since symmetric and asymmetric standard deviation (SSD and ASD) intervals were overlapped. However, the EE speed allowed to us distinguish between HRC and TO control.

The second approach, which is based on the box-and-whisker method, revealed differences in distance, \#MLLs, and time, as regards control methodologies, whereas differences in the user interfaces can be distinguished only by analyzing the robotic arm EE average speed. The box-and-whisker plots of the speed parameter are shown in Fig. 5.

These considerations were also confirmed by the results obtained from the $t$-test analysis, considering $p$-values equal to 0.01 and 0.005 .

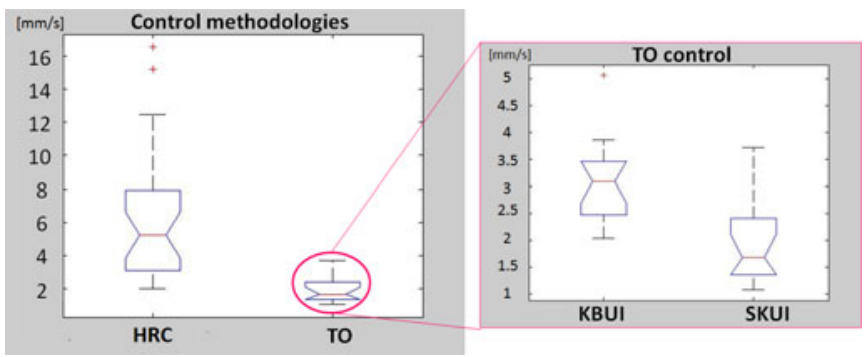

Fig. 5. Box-and-whisker plots of the robotic arm EE speed for the HRC and TO control user interfaces.

TABLE II

RESULTS OBTAINED USING THE ANOVA BETWEEN THE HRC AND TO CONTROL METHOdologies AND AS a COMBINATION OF THE F/T SENSOR, KBUI, AND SKUI

\begin{tabular}{|c|c|c|c|c|c|}
\hline & HRC-TO & KBUI-F/T & SKUI-F/T & KBUI-SKUI-F/T & KBUI-SKUI \\
\hline Marker [\%] & $7.7210^{-2}$ & $9.1210^{-2}$ & $1.210^{-1}$ & $2.08610^{-1}$ & $8.45910^{-1}$ \\
\hline Time [s] & $210^{-4}$ & $2.110^{-3}$ & $10^{-4}$ & $8.0210^{-5}$ & $4.3210^{-2}$ \\
\hline Distance [m] & $5.3810^{-7}$ & $410^{-4}$ & $4.8710^{-5}$ & $2.6610^{-6}$ & $1.22910^{-1}$ \\
\hline \#MLLs & $0.53810^{-7}$ & $210^{-3}$ & $4.610^{-3}$ & $2.6610^{-6}$ & $6.43610^{-1}$ \\
\hline EE speed $[\mathrm{mm} / \mathrm{s}]$ & $1.7710^{-12}$ & $310^{-4}$ & $1.5910^{-8}$ & $7.1810^{-12}$ & $4.5310^{-7}$ \\
\hline $\mathbf{f}_{s d X}[\mathrm{mHz}]$ & $610^{-4}$ & $2.910^{-2}$ & $5.8210^{-5}$ & $510^{-5}$ & $2.1810^{-3}$ \\
\hline $\mathbf{f}_{s d Y}[\mathbf{m H z}]$ & $2.9510^{-5}$ & $5.510^{-3}$ & $8.3110^{-5}$ & $2.4110^{-5}$ & $1.34410^{-2}$ \\
\hline
\end{tabular}

Values lower than 0.03 are reported in bold.

The ANOVA was the last and most detailed statistical analysis carried (threshold value equal to 0.03); results are reported in Table II. This analysis showed that the interfaces did not present relevant differences with respect to the percentage of identified markers. Moreover, the ANOVA suggested that differences regarding distance, \#MLLs, and time parameters were related to control methodologies. Likewise, ANOVA found significant statistical differences between interfaces as a function of the EE speed parameter.

The results for the percentage of identified markers deriving from the statistical analysis confirm that the robotic-aided platform allows accurate endoscopic procedures for all different control solutions, since the parameter has a high average value (i.e., around $80 \%$ ). These percentages are comparable with the results reported in [10] and [12].

Although statistical differences were found, the analysis of the elapsed time, distance, and \#MLLs parameters reveals that significant differences may only be found in the control methodologies. Although TO control results in a slower procedure, it provides more reliable magnetic link stability, resulting in lower capsule loss, compared with 
HRC control, as confirmed by the \#MLLs parameter. Therefore, the TO control could represent the more suitable methodology to enable a reliable capsule control and for more effective performance of the endoscopic procedure. Although the average speed parameter allows distinction between user interfaces, the selection of the optimal user interface should take into account parameters that are related to accuracy and procedure smoothness, rather than to speed only.

An estimation of the power spectral density of the signal was executed for the EE spatial $\mathrm{X}$ and $\mathrm{Y}$ trajectories, and the obtained $\mathrm{f}_{s d X}$ and $\mathrm{f}_{s d Y}$ parameters were processed with the same statistical approach. The bar charts statistical analysis did not show any differences between the control methodologies and interfaces $\left(\mathrm{f}_{s d X}\right.$ and $\mathrm{f}_{s d Y}$ in Table I), while the control methodologies were noticeable by means of the boxand-whisker and $t$-test approaches. The ANOVA allowed significant statistical differences to be detected between the control methodologies and between the different interfaces (see $\mathrm{f}_{s d X}$ and $\mathrm{f}_{s d Y}$ in Table II). Therefore, based on the operating assumption, the results obtained by the analysis of the $\mathrm{f}_{s d X}$ and $\mathrm{f}_{s d Y}$ parameters confirmed TO control methodology and emphasized how the serial kinematic haptic interface could be the most suitable user interface to perform successful endoscopic procedures, since it allows smoother and primed robotic arm motion trajectories and, consequently, more reliable and effective control of the capsule.

\section{CONCLUSION}

An HRC control and a TO control has been implemented on a recently developed endoscopic platform, thus enabling robotic-aided magnetic locomotion for an endoscopic capsule in traditional diagnostic procedures of the GI tract, such as colonoscopy. An experimental ex vivo protocol was carried out in collaboration with a team of 15 endoscopists to compare performance between different control methodologies and user interfaces. All clinicians successfully completed the ex vivo task, showing promising results in terms of procedure accuracy and reliability. Several parameters were measured in each experimental trial and a statistical analysis was performed, in order to derive the most adequate solution for capsule motion control during endoscopic procedures. The statistical analysis of the control parameters established that TO control is more reliable than HRC control; furthermore, a more detailed analysis on the robotic arm variation frequency parameter demonstrated that, in a TO scenario, an SKUI is a more suitable control interface to perform smooth robotic-aided endoscopic procedures.

The proposed study, which is based on the detailed statistical analysis of significant control parameters, could represent a proper and general approach for the evaluation of the more suitable control methodology. Findings could be generalized to robotic-aided magnetic-based platforms for capsule endoscopy, as in [13]-[15].

\section{ACKNOWLEDGMENT}

The authors would like to thank KARL STORZ GmbH and Co. $\mathrm{KG}$, Tuttlingen, Germany, for providing the vision system and the medical doctors involved in the experimental tests from the Center for Minimally Invasive Surgery, University of Turin, Turin, Italy.

\section{REFERENCES}

[1] S. C. Low and L. Phee, "A review of master-slave robotic systems for surgery," in Proc. IEEE Conf. Robot., Autom., Mechatronics, 2004, pp. 3742.

[2] H. Taylor and D. Stoianovici, "Medical robotics in computer-integrated surgery," IEEE Trans. Robot. Autom., vol. 19, no. 5, pp. 765-781, Oct. 2003.

[3] P. Dario, B. Hannaford, and A. Menciassi, "Smart surgical tools and augmenting devices," IEEE Trans. Robot. Autom., vol. 19, no. 5, pp. 782792 , Oct. 2003.

[4] Stereotaxis website. (2011). [Online]. Available: http://www.stereotaxis. com.

[5] P. Dario, P. Ciarletta, A. Menciassi, and B. Kim, "Modeling and experimental validation of the locomotion of endoscopic robots in the colon," Int. J. Robot. Res., vol. 23, pp. 549-556, 2004.

[6] A. Moglia, A. Menciassi, P. Dario, and A. Cuschieri, "Capsule endoscopy: Progress update and challenges ahead," Nat.Rev. Gastroenterol. Hepatol., vol. 6, pp. 353-61, 2009.

[7] P. Valdastri, R. J. Webster, C. Quaglia, M. Quirini, A. Menciassi, and P. Dario, "A new mechanism for mesoscale legged locomotion in compliant tubular environments," IEEE Trans. Robot., vol. 25, no. 5, pp. 104757, Oct. 2009.

[8] G. D. Hager, A. M. Okamura, P. Kazanzides, L. L. Whitcomb, G. Fichtinger, and R. H. Taylor, "Surgical and interventional roboticsPart III: Surgical assistance systems," IEEE Robot. Autom. Mag., vol. 15, no. 4, pp. 84-93, Dec. 2008.

[9] G. Ciuti, P. Valdastri, A. Menciassi, and P. Dario, "Robotic magnetic steering and locomotion of capsule endoscope for diagnostic and surgical endoluminal procedures," Robotica, vol. 156, pp. 48-58, 2009.

[10] G. Ciuti, R. Donlin, P. Valdastri, A. Arezzo, A. Menciassi, M. Morino, and P. Dario, "Robotic versus manual control in magnetic steering of an endoscopic capsule," Endoscopy, vol. 42, pp. 148-52, 2010.

[11] B. Mitchell, J. Koo, I. Iordachita, P. Kazanzides, A. Kapoor, J. Handa, G. Hager, and R. Taylor, "Development and application of a new steadyhand manipulator for retinal surgery," in Proc. IEEE Int. Conf. Robot. Autom., 2007, pp. 623-629.

[12] J. E. Garbus, J. J. Singh, and The SAGES Colonoscopy Outcomes Study Group, "A prospective analysis of 13580 colonoscopies," Surg. Endosc., vol. 15, no. 3, pp. 251-261, 2001.

[13] F. Carpi, N. Kastelein, M. Talcott, and C. Pappone, "Magnetically controllable gastrointestinal steering of video capsules," IEEE Trans. Biomed. Eng., vol. 58, no. 2, pp. 231-234, Feb. 2011.

[14] J. F. Rey, H. Ogata, N. Hosoe, K. Ohtsuka, N. Ogata, K. Ikeda, H. Aihara, I. Pangtay, T. Hibi, S. Kudo, and H. Tajiri, "Feasibility of stomach exploration with a guided capsule endoscope," Endoscopy, vol. 42, pp. 541-545, 2010 .

[15] M.-G. Kim, Y.-S. Hong, and E.-J. Lim, "Position and orientation detection of capsule endoscopes in spiral motion," Int. J. Precis. Eng. Manuf., vol. 11, no. 1, pp. 31-37, 2010. 